\title{
HMGA2 promotes colorectal cancer angiogenesis via dual regulation of Sema3A and VEGFA
}

\author{
Cancan Wang ${ }^{1}$, Yi Long ${ }^{1}$, Miaomiao Tao ${ }^{1}$, Hongbo $\mathrm{Ma}^{1}$, Yanyan $\mathrm{Li}^{1}$, Gendou Zhou ${ }^{1}$, Xiaoqiao Hu${ }^{1}$, Wei Wang ${ }^{1}$, Ruyan Liu ${ }^{1}$, Meiyu \\ Zhou ${ }^{1}$, Guofa Xu ${ }^{1}, \quad$ Zexin Wang ${ }^{1}$, Min Chen ${ }^{*}$, Qi Zhou ${ }^{1 *}$ \\ 1 Fuling Central Hospital, Chongqing China 400038 \\ *Corresponding Author: 2099780278@qq.com (M.C.); qizhou112@163.com (Q.Z.)
}

\begin{abstract}
Background: HMGA2 encodes a small non histone chromatin-associated protein that has no intrinsic transcriptional activity, but can modulate transcription by altering the chromatin architecture. HMGA2 was found overexpressed in a variety of epithelial and mesenchymal tumors and promoted invasion and metastasis in most malignant epithelial tumors. A recent study showed that P53 inhibited CRC progression by targeting HMGA2. However, the mechanism by which HMGA2 affect angiogenesis in CRC has not been clarified. Methods: The expression of HMGA2 was analyzed by IHC, WB and bio infomatic analysis. Cbioportal and mexpress online tools were applied to explore the CNV and methylation of HMGA2 in CRC patients. Single cell data from GEO was used to examine the specific cell type that contribute to the high HMGA2 expression in CRC. Lentivirus was used to knock down HMGA2 in CRC cells and HUVECs was used to study angiogenesis. Results: In the current study, we first detected the expression pattern of HMGA2 in CRC patients and evaluated its clinical values and CNV amplification could possibly contribute to the up regulation of HMGA2 in CRC patients. By analyzing CRC single cell data we found that HMGA2 was specifically up regulated in the colorectal epithelial cells. Furthermore, knocking down of HMGA2 suppresses angiogenesis via dual regulation of VEGF-A and SEMA3A in CRC through inactivating VEGRR2 pathway in HUVECs. Conclusions: HMGA2 might be a promising prognostic marker and target for treating advanced CRC patients.
\end{abstract}

Key words: HMGA2; Colorectal cancer; Sema3A; VEGFA

\section{Introduction}

Colorectal cancer (CRC) is the fourth most deadly cancer with almost 900000 deaths annually [1]. Although the survival outcome of CRC patients has dramatically increased due to the improvement of diagnostic and therapeutic strategies, up to $20 \%$ of CRC patients indicate 
metastatic phenotype upon initial diagnosis, and at least $30 \%$ them will die of metastatic cancer[2], thus make the advanced CRC patients suffer from undesired 5-year survival rate(lower than $10 \%)[3,4]$.

The resistance to traditional radiotherapy and chemotherapy was the main reason of the worse prognosis of advanced CRC. Therefore, it is very important to develop novel therapeutic strategies that may improve survival of the advanced CRC. Tumor angiogenesis provides $\mathrm{O}_{2}$ and nutrients to tumor cells and is widely believed to be one the of most important control points of tumor invasion and metastasis, which is responsible for the high mortality and poor prognosis [4, 5]. As long as tumor size is less than $2 \mathrm{~mm}$ in diameter, inhibiting angiogenesis can prevent further growth and metastasis[5-7]. Anti-angiogenic treatments have been widely used for CRC treatment. But the adverse effects of increased risk of stroke and arterial events, gastrointestinal bleeding, gastrointestinal perforation, delayed wound healing was increase after use anti-angiogenesis therapy was confined with its side effects $[8,9]$. Therefore new therapeutic strategies of tumor angiogenesis would be needed to improve patient survival.

Carcinogenesis is the pathological alteration of epithelial/mesenchymal cells under the stimulation of carcinogenic factors, including inflammation, chemicals, and radiation [10] . HMGA2 encodes a small non histone chromatin-associated protein that has no intrinsic transcriptional activity, but can modulate transcription by altering the chromatin architecture $[11,12]$. HMGA2 was found overexpressed in a variety of epithelial and mesenchymal tumors [13-15] and promoted invasion and metastasis in most malignant epithelial tumors [16,17]. A recent study showed that P53 inhibited CRC progression by targeting HMGA2[18] However, the mechanisms by which HMGA2 affect angiogenesis in CRC has not been clarified.

In the current study, we first detected the expression pattern of HMGA2 in CRC patients and evaluated its clinical values and CNV amplification could possibly contribute to the up regulation of HMGA2 in CRC patients. By analyzing CRC single cell data we found that HMG2 was specifically up regulated in the colorectal epithelial cells. Furthermore, knocking down of HMGA2 suppresses angiogenesis via dual regulation of VEGF-A and SEMA3A in CRC through inactivating VEGRR2 pathway in HUVECs. 


\section{Results}

\subsection{HMGA2 is overexpressed due to copy number amplification in CRC patients and} associates with worsen prognosis

To investigate the potential role of HMGA2 in colorectal cancer, we first detected the expression pattern of HMGA2 in CRC patients' sample and cell lines. HMGA2 expression was markedly higher in CRC patients' sample and cell lines as detected by IHC and WB (Fig. 1A, B ,D) and this observation was further confirmed by analyzing the TCGA colorectal cancer cohort (Fig. 1C). Thus we speculated whether this abnormal expression could reflect clinical progression. We collected 154 CRC patients' sample with clinical information and found that patients featured by low differentiation and advanced clinical stage showed high expression of HMGA2(Fig. 2 A, B) which was further confirmed using public datasets (Fig. 2C). High HMGA2 expression showed worse prognosis $(\mathrm{P}<0.05$; Fig. 1D, E). Tumor angiogenesis is a vital supporter of tumor invasion and metastasis, which is responsible for the high mortality and poor prognosis[19]. The expression of CD31 could partially reflect the formation of micro vascular and denoted increased angiogenesis [18] and we found that HMGA2 protein level was positively correlated with CD31 expression in serial sections of CRC tissues from 154 cases and TCGA CRC datasets (Fig. 2F).

Due to the heterogeneity origins of CRC [20] we analyzed a CRC single cell data set (including 9 CRC patient samples) to unveil which type of cell contribute to the high expression of HMGA2. After tSNE reduction and cell type annotation (Fig. 3A, B) we found that colorectal epithelial cells showed high expression of HMGA2 among 7 types of cells (Fig. 3C). To further investigate the possible mechanisms contributed to the abnormal expression of HMGA2 in CRC. We analyzed online data sets and found CNV was significantly disrupted in CRC (Fig. 4A) and gain of copy number denoted high expression of HMGA2 by analyzing TCGA data sets (Fig. 4 B-D), even though promoter methylation was observed however it was not correlated with gene expression(Fig. 4E). Thus the above results manifested that overexpression of HMGA2 was associated with undesired disease progression which could possible attribute to angiogenesis. 


\subsection{HMGA2 knock down inhibits CRC induced HUVECs metastasis in vitro and tumor} formation in vivo

Previous study on HMGA2 in CRC was confined to CRC cells per se $[21,22]$. However, accumulating studies emphasized the micro environment in modulating the progression of tumors [23]. Thus we collected the Conditional medium (CM) from scrambled or HMGA2 shRNA lentivirus infected cells and treated HUVECs as previously described [3]. The results showed that HMGA2 shRNA CM from two CRC cell lines (Fig. 5A, B) could both inhibit the invasion $\mathrm{m}$ and migration (Fig. 5C, D) of HUVECs. To further confirm the effects of HMGA2 knock down on tumor formation in vivo, HCT-116 NC and HMGA2 KD cells were subcutaneously transplanted to nude mice. After 20 days, mice were euthanized and tumors were harvested, weighed, and analyzed (Fig.6). The results showed that HMGA2 konck down reduced the tumor formation (Fig. 6A, B). Tumor angiogenesis was determined by CD31 expression in tumor specimens through IHC and IF. The result showed that HMGA2 KD inhibited tumor angiogenesis in mice (Fig. 6C, D). Collectively, these results indicated that knock down HMGA2 in CRC cells could inhibit HUVECs metastasis and tumor formation.

\subsection{Knock down of HMGA2 suppresses angiogenesis via dual regulation of VEGF-A and}

\section{SEMA3A in CRC through inactivating VEGRR2 pathway in HUVECs.}

Since the CM could accelerate the metastasis and invasion of HUVECs, we next tried to unveil the possible signal molecule involved in this process. We firstly detected the levels of angiogenesis related secreted factors: HGF, Endothelin-1, angiostatin, VEGF-A, VEGF-C, Sema3A and PDGF-BB in the supernatant of HMGA2 KD and NCHCT-116 and HMGA2 KD and NC HT-29 cells. While HGF, Endothelin-1, angiostatin, VEGF-C and PDGF-BB were not changed significantly (Fig. 7A), VEGF-A level was lower and Sema3A level was higher in the supernatant (Fig. 7B, C). Previous studies showed that VEGF-A was a major factor that regulates angiogenesis by activating the tyrosine kinase receptor VEGFR-2[24], and SEMA3A competitively bond to VEGFR-2 to suppress tumor angiogenesis[25]. Then we detected the expression of VEGFA and Sema3A in CRC patient tissues. We found VEGF-A level was lower while SEMA3A was up-regulated in HMGA2 low expression patients vice versa (Fig. 7D). We further found that HMGA2 was positively correlated with VEGFA and negatively correlated with Sema3A when analyzing TCGA COAD data sets (Fig. 7E). 
To confirm the direct regulation of HMGA2 on VEGFA and SMA3A, we analyzed the protein expression by knocking down HMGA2 in HCT116 cells. Since previous study showed that HMGA2 could repress TGF $\beta$ signaling [26] we first confirmed this observation in our model (Fig. $8 \mathrm{~A})$ and subsequently we found that HMGA2 knock down up regulated SEMA3A and inhibited VEGFA(Fig. 8A). Based on our observation that HMGA2 KD increased VEGF-A and reduced SEMA3A levels in the supernatant of HCT-116 and HT-29 colon cancer cells, we evaluated the activation of VEGFR2 pathway in HUVECs by detecting total and phosphorylation expression of VEGF-R2, ERK, and Akt in HUVECs which stimulated with conditioned medium. We found that HMGA2 KD conditioned medium from HCT-116 decrease the phosphorylation protein expression of VEGFR-2, ERK, and Akt, which are important regulator of endothelial cell function such as cell migration, endothelium-dependent relaxation, and angiogenesis (Fig. 8B). These data suggested that HMGA2 promoted angiogenesis in CRC by enhancing VEGFA and suppressing SEMA3A simultaneously, which activated VEGFR-2 on the surface of HUVECs to promote metastasis.

\section{Materials and Methods}

\subsection{Patient samples}

Surgically resected specimens were collected from 154 CRC cases and paired adjacent normal tissues at Third Military Medical University and Fu Ling Central Hospital from 2004 to 2007. The specimens were fixed with $4 \%$ neutral buffered paraformaldehyde and embedded in paraffin; after staining with hematoxylin and eosin, the specimens were analyzed by a senior pathologist. A semi-automated tissue microarrayer (Beecher Instruments, Sun Prairie, WI, USA) was used to construct tissue microarrays (needle diameter: $1 \mathrm{~mm}$ ) by punching out one or two tissue cores from each specimen to obtain two paraffin blocks containing 154 CRC specimens and paired adjacent normal tissues. The study protocol was approved by the Ethical Committee of Fuling Central Hospital (NO. 2019-05-05) and Third Military Medical University (NO. 2017-03-24) and the research conformed to the principles of the Declaration of Helsinki.

\subsection{Cell lines}

Normal colon epithelial cell line FHC and human umbilical vein endothelial cells(HUV-EC-Cs) were purchased from the American Type Culture Collection (Manassas, VA, 
USA).HCT-116, LS174T, HT-29, SW480, SW620, RKO, LoVo, HCT-8 and Caco2 CRC cell lines were purchased from Cell Bank of Shanghai Institute of Cell Biology, Chinese Academy of Sciences. LS174T, RKO, and Caco2 cells were cultured in Eagle's Minimum Essential Medium; FHC cells were cultured in Dulbecco's Modified Eagle's Medium:F12 (Gibco); HCT-116 and HT-29 and HCT-8 cells were cultured in McCoy's 5a medium; SW480 and SW620 cells were cultured in Leibovitz's L-15 medium; and HUV-EC-Cs and LoVo cells were cultured in F-12K medium. All media were purchased from Gibco (Grand Island, NY, USA) and were supplemented with $10 \%$ fetal bovine serum (FBS) (Gibco). Cells were cultured at $37^{\circ} \mathrm{C}$ and $5 \% \mathrm{CO}_{2}$.

\subsection{Immunohistochemistry}

Formalin-fixed, paraffin-embedded tissue specimens and xenografts were cut into 5 - $\mu$ m-thick sections that were analyzed by immunohistochemistry using the REAL EnVision Detection System (Dako, Carpinteria, CA, USA) according to the manufacturer's protocol. The following primary antibodies were used: rabbit monoclonal anti-HMGA2 (1:2000; OriGene, Rockville, MD, USA), rabbit polyclonal anti-cluster of differentiation (CD31) (1:500; Abcam, Cambridge, MA, USA), rabbit polyclonal anti-VEGF (1:1000; Abcam), and rabbit polyclonal anti-SEMA3A (1:500; Abcam). The percentage of immune positive cells (staining percentage) was determined under the microscope and scored as follows: 0 , no positive cells; $1,1 \%-25 \%$ positive cells; $2,26 \%-50 \%$ positive cells; $3,51 \%-75 \%$ positive cells; and $4, \geq 76 \%$ positive cells. Intensity was scored as $0=$ none, $1=$ weak, $2=$ moderate, and $3=$ strong. Comprehensive score $=$ staining percentage $\times$ intensity. HMGA2 expression was classified into low/ high expression group according to the median of total samples.

\subsection{Western blotting}

WB was performed as previously described. The primary antibodies used in this study were: rabbit monoclonal anti-HMGA2 (1:2000; OriGene);rabbit polyclonal anti-SEMA3A (1:1000) and rabbit monoclonal anti-Smad2 (1:1000), rabbit monoclonal anti-Smad3, rabbit monoclonal anti-TGF $\beta(1: 1000)$ (1:1000) (both from Abcam); and rabbit monoclonal anti-VEGFR-2 (1:1000), rabbit monoclonal anti-phosphorylated (p-)VEGFR-2(Y1175)(1:1000), rabbit monoclonal anti-extracellular signal-regulated kinase (ERK)1/2 (1:1000), rabbit monoclonal anti-p-ERK1/2 (1:1000), rabbit monoclonal anti-Akt (1:1000), rabbit monoclonal anti-p-Akt (1:1000) and rabbit polyclonal anti- $\beta$-actin (1:3000) all from Cell Signaling Technology; Danvers, MA, USA). 


\subsection{Plasmid construction and stable transfection of cell lines}

HMGA2 short hairpin (shRNA) lentiviral particles were constructed by Genechem Co. (Shanghai, China). The shRNA sequence targeting HMGA2 and a non-targeting scrambled sequence were as follows: shHMGA2\#1, GAAATGGCCACAACAAGTTGT; shHMGA2\#2, AGTCCCTCTAAAGCAGCTCAA; shHMGA2\#3, TCCTCACAAGAGTCTGCCGAA. HCT-116 and HT-29 cells were cultured in 24-well plates at $5 \times 10^{4} /$ well and infected with lentivirus containing the shRNA constructs for $24 \mathrm{~h}$ without FBS. Fresh culture medium containing $1 \mu \mathrm{g} / \mathrm{ml}$ puromycin was added to select stably transfected cell lines. The knockdown (KD) efficiency of HMGA2 was confirmed by western blotting.

\subsection{Conditioned medium preparation and treatment for HUVECs}

Equal numbers of HCT-116 and HT-29 cells infected with lentivirus containing scrambled or HMGA2 shRNA were seeded in $100-\mathrm{mm}$ dishes and allowed to attach. The medium was centrifuged at $1000 \mathrm{rpm}$ for 3 min to remove any cell contaminants and termed as Conditional medium (CM). As for the treatment of HUVECs, CM collected from scrambled or HMGA2 shRNA HCT116 cells were subsequently subjected to the culture of HUVECs.

\subsection{Proliferation, migration, invasion}

HUVECs proliferation was analyzed after cultured in $\mathrm{CM}$ for $0,6,12,24$, and $36 \mathrm{~h}$ using Cell Counting Kit-8 (Dojindo Laboratories, Kumamoto, Japan) according to the manufacturer's protocol. Briefly, $2 \times 10^{5} \mathrm{HUV}$-EC-C cells were seeded in 24 -well plate with complete medium for $8 \mathrm{~h}$ and subsequently cultured in serum-free medium. CM was added respectively and wound closure (migration) was evaluated at 0 and $24 \mathrm{~h}$. As for invasion assay, the $8.0-\mu \mathrm{m}$ pore transwell membrane was coated with $1 \mathrm{mg} / \mathrm{ml}$ Matrigel (BD Biosciences, Franklin Lakes, NJ, USA). The lower chamber was filled with F-12K medium containing 10\% FBS and the upper chamber was filled with a cell suspension containing $1 \times 10^{4}$ cells in $\mathrm{CM}$. Cells were allowed to migrate at $37^{\circ} \mathrm{C}$ and $5 \% \mathrm{CO}_{2}$ for $48 \mathrm{~h}$. Invasion cells were fixed and stained with crystal violet and counted under a light microscopy in five randomly selected fields. Results were expressed as the average number of cells per field. The experiments were performed three times independently.

\subsection{In vivo tumor formation assay}

Male nude mice (4 to 6 weeks old) were purchased from the Center of Experimental Animals 
of Third Military Medical University (Chongqing, China). The mice were subcutaneously inoculated with HCT-116 cells $\left(1 \times 10^{6}\right)$ infected with lentivirus expressing HMGA2 or scrambled shRNA. Tumor-bearing mice were euthanized 3 weeks after transplantation. The width and length of tumors were measured with calipers, and tumor volume was calculated according to the formula: volume $\left(\mathrm{mm}^{3}\right)=$ width $(\mathrm{mm})^{2} \times$ length $(\mathrm{mm}) \times 0.4$. All experimental animal procedures were approved by the Institutional Animal Care and Use Committee of Third Military Medical University, China and followed the principles of the Declaration of Helsinki.

\subsection{Immunofluorescence microscopy}

Frozen sections of fresh xenograft tissue were fixed with 4\% paraformaldehyde and treated with triton-100 and then incubated with rabbit polyclonal anti-CD31 (1:500) and rabbit monoclonal anti-HMGA2 $(1: 2000)$ antibodies followed by the secondary antibodies. Nuclei were counterstained with DAPI (Sigma-Aldrich, St. Louis, MO, USA), and slides were visualized with a laser confocal scanning microscope (SP-5; Leica Microsystems, Wetzlar, Germany).

\subsection{Enzyme-linked immunosorbent assay (ELISA)}

Secretion of VEGF-A, angiostatin (ANG)-2, VEGF-C, hepatocyte growth factor (HGF), Endothelin-1, PDGF-BB and SEMA3A was determined using ELISA kits (Senxiong Biotech, Beijing, China) according to the manufacturer's instructions and normalized to the total number of cells.

\subsection{Bioinformatics analysis and public data base}

TCGA COAD raw counts data was download use GDC client from https://portal.gdc.cancer.gov/. Raw data was processed in R (version 4.0.2) using Dseq2 and EdgeR package and figures were plotted using ggplot 2 .

Single cell analysis: The single cell data set of 9 CRC patients was retrieved from GEO GSE166555 and standard pipeline was applied for data processing using R. All clusters of cells in CRC were annotated by singleR and CellMarker according to the composition of the marker genes.

\subsection{Statistical analysis}

Statistical analyses were performed using SPSS v.17.0 software (SPSS Inc., Chicago, IL, USA). Kaplan-Meier survival plots and the log-rank test were used to compare patient survival rates. Differences between experimental groups and controls were assessed with the Student's test. 
$\mathrm{P}<0.05$ was considered statistically significant.

\section{Discussion}

Angiogenesis is the fundamental step of tumors transition from dormant to malignant state and it is recognized as one of the hallmarks of cancer. since its critical role in tumor progression, invasion, and metastasis; therefore, angiogenesis represents a rational target for therapeutic intervention[27]. A recent study showed epigenetic regulation contributed to the expression of HMGA2 in COAD [28], however our data for the first time indicated that CNV was another factor in regulating HMGA2 expression in COAD. Different strategies for angiogenesis intervention are based on modulating any of the key steps of the angiogenic process, including endothelial cell proliferation, protease secretion, cell-matrix adhesion, migration, and invasion. Rooted in the belief that blocking vessel supply starves tumors to death[29], it has become increasingly accepted that blocking tumor angiogenesis as much as possible would provide cancer patients with maximal survival benefit. It is well knowns that vascular endothelial growth factor (VEGFA) inducing tumor angiogenesis [30], it is a secreted factor via banding with tyrosine kinase VEGF receptor 2 (VEGFR2) on the vascular endothelial cells. Indeed, the monoclonal anti-VEGF antibody bevacizumab [31,32] and the second-generation multitargeted receptor tyrosine kinase inhibitors (RTKIs) sunitinib [33] and sorafenib [34] have prolonged the life of numerous cancer patients. However, drug resistance would be inevitably established out of unknown mechanisms. Thus, uncover novel pathways in CRC would provide potential target to inhibit tumor angiogenesis.

HMGA2 is an architectural transcription factor or organize assembly on enhancers of a variety of genes and overexpressed in a variety of epithelial and mesenchymal tumors. Since angiogenesis was closely related to metastasis, HMGA2 promotes invasion and metastasis in most malignant epithelial tumors. We first analyzed the expression of HMGA2 in colorectal cancer. Our experiments confirmed that HMGA2 was over expressed in human colorectal cancer cell and patient tissue, which also associated with poor prognosis. However, the relationship between HMGA2 and angiogenesis in CRC has not been previously reported. In the current study HMGA2 was found positively correlated with the density of CD31 in colorectal cancer patient tissues. Furthermore, we established HMGA2-deficient HCT-116 and HT-29 cell lines and conditioned medium from the HMGA2 KD and scramble cells were applied to culture HUVECs. We found that the conditioned medium from negative controls cells promote the migration, invasion while 
HMGA2 KD inhibited theses effects. Furthermore, we showed that HMGA2 promoted CRC angiogenesis by dual regulating Sema3A and VEGFA. It is well known that VEGFA inducing tumor angiogenesis via banding with tyrosine VEGFR2 on the vascular endothelial cells[35] and SEMA3A can inhibit the binding of VEGFA to NP1 which is able to inhibit in vitro angiogenesis[25]. Thus, HMGA2 might be a promising target due to its multiple roles in compromising CRC angiogenesis. 
Figure legends

\section{Figure 1}

Figure 1. HMGA2 is over expressed in colorectal cancer

A: Representative IHC staining of HMGA2 the colorectal cancer and adjacent normal colorectal tissues; B: Statistical analysis on IHC staining scores based on quantification of HMGA2 expression in colon tumor and adjacent normal colon tissues. ( $\mathrm{n}=154$ for each group); $\mathrm{C}$ : The expression pattern of HMGA2 in colorectal cancer was further confirmed using TCGA cohort (Normal = 41, Tumor=472; $\mathrm{P}<0.01$ ); D: HMGA2 expression was detected in normal and colon tumor cell lines

Figure 2

Figure 2. High HMGA2 expression is associated with disease progression. A-B HMGA2 expression was positive correlated with CRC clinical progression in our samples; C: HMGA2 protein level was positive correlated with CRC clinical progression using TCGA data retrieved from cbioportal (CPTAC-2 Prospective Cell 2019); D: Statistical analysis of the correlation between HMGA2 expression levels and overall survival (OS) of CRC patients (Kaplan-Meier Survival Curves) from tissue microarray. ( $\mathrm{P}<0.05)$; E: High expression of HMGA2 denoted poor survival as shown by GEO data (GSE17537); F: HMGA2 expression was positively correlated with CD31 expression level (PECAM: CD31).

Figure 3

Figure 3. HMGA2 is preferably overexpressed in colorectal epithelial cell in CRC patients

A: The tSNE algorithm was applied for dimensionality reduction of $9 \mathrm{CRC}$ patients( data was retrieved from GEO: GSE166555); B: All clusters of cells in CRC were annotated by singleR and CellMarker according to the composition of the marker genes; C: HMGA2 expression was up regulated specifically in colorectal epithelial cells.

Figure 4

Figure 4. Copy number amplification rather promoter methylation contribute to the overexpression of HMGA2 in COAD patients

A: HMGA2 CNV was significantly amplified in CRC as analyzed using mexpress (https://mexpress.be/index.html); B-D: HMGA2 expression was correlated to CNV as analyzed using cbioportal online tools (B: TCGA, PanCancer Atlas; C: CPTAC-2 Prospective Cell 2019; D: 
TCGA Nature 2012); E: HMGA2 expression was not correlated with methylation status (TCGA Nature 2012).

Figure 5

Figure 5. Conditional medium of HMGA2 Knockdown CRC cells inhibits HUVECs metastasis in vitro

A-B: HMGA2 knockdown efficiency was examined in two colon cancer epithelia cells;

C-D: HMGA2 knockdown conditional medium significantly inhibited migration of HUVECs.

Figure 6

Figure 6. Knockdown of HMGA2 inhibits tumor growth and angiogenesis in vivo A-B: HMGA2 knockdown reduce tumor formation;

C-D: HMGA2 knockdown reduced CD31 expression as detected by tissue IHC and IF.

\section{Figure 7}

Figure 7. Knockdown of HMGA2 increase VEGFA and decrease Sema3A secretion in colon rectal epithelial cells conditional medium

A: Angiogenesis related factors were examined in two colon rectal epithelial cells knock down conditional medium which showed no significant change;

B-C: Sema3A and VEGFA were deregulated in two colon rectal epithelial cells knock down conditional medium;

D: VEGFA expression was positively while SEMA3A was negatively correlated with HMGA2 expression in our cohort as manifested by IHC;

E: The correlations between HMGA2 and SEMA3A (up) or VEGFA (down) were further confirmed by TCGA CRC cohort.

Figure 8

Figure 8. Knockdown of HMGA2 activated the VEGFR2-ERK-AKT signaling in HUVECs

A: Knockdown of HMGA2 in CRC cells inhibited VEGFA and up regulated SEMA3A;

B: HMGA2 knockdown conditional medium inhibited VEGFR2- Akt - ERK signaling in HUVEC. 
1. Dekker, E.; Tanis, P.J.; Vleugels, J.L.A.; Kasi, P.M.; Wallace, M.B. Colorectal cancer. Lancet 2019, 394, 1467-1480, doi:10.1016/S0140-6736(19)32319-0.

2. Mody, K.; Baldeo, C.; Bekaii-Saab, T. Antiangiogenic Therapy in Colorectal Cancer. Cancer journa/2018, 24, 165-170, doi:10.1097/PPO.0000000000000328.

3. Deng, F.; Zhou, R.; Lin, C.; Yang, S.; Wang, H.; Li, W.; Zheng, K.; Lin, W.; Li, X.; Yao, X.; et al. Tumor-secreted dickkopf2 accelerates aerobic glycolysis and promotes angiogenesis in colorectal cancer. Theranostics 2019, 9, 1001-1014, doi:10.7150/thno.30056.

4. Redman, J.M.; Rhea, L.P.; Brofferio, A.; Whelpley, M.; Gulley, J.L.; Gatti-Mays, M.E.; McMahon, S.; Cordes, L.M.; Strauss, J. Successful 5-fluorouracil (5-FU) infusion re-challenge in a metastatic colorectal cancer patient with coronary artery disease who experienced symptoms consistent with coronary vasospasm during first 5-FU infusion. Journal of gastrointestinal oncology 2019, 10, 1010-1014, doi:10.21037/jgo.2019.07.04.

5. Ntellas, P.; Dadouli, K.; Perivoliotis, K.; Sogka, E.; Pentheroudakis, G.; loannou, M.; Hadjichristodoulou, C.; Tepetes, K.; Mauri, D. Microvessel Density and Impact of Angiogenesis on Survival of Resected Pancreatic Cancer Patients: A Systematic Review and Meta-analysis. Pancreas 2019, 48, 233-241, doi:10.1097/MPA.0000000000001237.

6. Saravanan, S.; Vimalraj, S.; Pavani, K.; Nikarika, R.; Sumantran, V.N. Intussusceptive angiogenesis as a key therapeutic target for cancer therapy. Life sciences 2020, 252, 117670, doi:10.1016/j.Ifs.2020.117670.

7. Wang, Z.; Dabrosin, C.; Yin, X.; Fuster, M.M.; Arreola, A.; Rathmell, W.K.; Generali, D.; Nagaraju, G.P.; El-Rayes, B.; Ribatti, D.; et al. Broad targeting of angiogenesis for cancer prevention and therapy. Semin Cancer Biol 2015, 35 Suppl, S224-S243, doi:10.1016/j.semcancer.2015.01.001.

8. Ebos, J.M.; Lee, C.R.; Cruz-Munoz, W.; Bjarnason, G.A.; Christensen, J.G.; Kerbel, R.S. Accelerated metastasis after short-term treatment with a potent inhibitor of tumor angiogenesis. Cancer Cel/2009, 15, 232-239, doi:10.1016/j.ccr.2009.01.021.

9. Loges, S.; Mazzone, M.; Hohensinner, P.; Carmeliet, P. Silencing or fueling metastasis with VEGF inhibitors: antiangiogenesis revisited. Cancer Cell 2009, 15, 167-170, doi:10.1016/j.ccr.2009.02.007.

10. Grivas, P.D.; Melas, M.; Papavassiliou, A.G. The biological complexity of urothelial carcinoma: Insights into carcinogenesis, targets and biomarkers of response to therapeutic approaches. Seminars in cancer biology 2015, 35, 125-132, doi:10.1016/j.semcancer.2015.08.006.

11. Cleynen, I.; Van de Ven, W.J. The HMGA proteins: a myriad of functions (Review). International journal of oncology 2008, 32, 289-305.

12. Fedele, M.; Battista, S.; Kenyon, L.; Baldassarre, G.; Fidanza, V.; Klein-Szanto, A.J.; Parlow, A.F.; Visone, R.; Pierantoni, G.M.; Outwater, E.; et al. Overexpression of the HMGA2 gene in transgenic mice leads to the onset of pituitary adenomas. Oncogene 2002, 21, 3190-3198, doi:10.1038/sj.onc.1205428.

13. Guo, L.; Chen, C.; Shi, M.; Wang, F.; Chen, X.; Diao, D.; Hu, M.; Yu, M.; Qian, L.; Guo, N. Stat3-coordinated Lin-28-let-7-HMGA2 and miR-200-ZEB1 circuits initiate and maintain oncostatin M-driven epithelial-mesenchymal transition. Oncogene 2013, 32, 5272-5282, doi:10.1038/onc.2012.573. 
14. Wang, X.; Liu, X.; Li, A.Y.; Chen, L.; Lai, L.; Lin, H.H.; Hu, S.; Yao, L.; Peng, J.; Loera, S.; et al. Overexpression of HMGA2 promotes metastasis and impacts survival of colorectal cancers. Clin Cancer Res 2011, 17, 2570-2580, doi:10.1158/1078-0432.CCR-10-2542.

15. Raskin, L.; Fullen, D.R.; Giordano, T.J.; Thomas, D.G.; Frohm, M.L.; Cha, K.B.; Ahn, J.; Mukherjee, B.; Johnson, T.M.; Gruber, S.B. Transcriptome Profiling Identifies HMGA2 as a Biomarker of Melanoma Progression and Prognosis. J Invest Dermato/ 2013, 133, 2585-2592, doi:10.1038/jid.2013.197.

16. Zhao, X.P.; Zhang, H.; Jiao, J.Y.; Tang, D.X.; Wu, Y.L.; Pan, C.B. Overexpression of HMGA2 promotes tongue cancer metastasis through EMT pathway. Journal of translational medicine 2016, 14, doi:ARTN 26

10.1186/s12967-016-0777-0.

17. Li, Y.; Zhao, Z.X.; Xu, C.H.; Zhou, Z.Q.; Zhu, Z.; You, T.G. HMGA2 induces transcription factor Slug expression to promote epithelial-to-mesenchymal transition and contributes to colon cancer progression. Cancer Lett 2014, 355, 130-140, doi:10.1016/j.canlet.2014.09.007.

18. Chen, X.; Zeng, K.; Xu, M.; Liu, X.; Hu, X.; Xu, T.; He, B.; Pan, Y.; Sun, H.; Wang, S. P53-induced miR-1249 inhibits tumor growth, metastasis, and angiogenesis by targeting VEGFA and HMGA2. Cell death \& disease 2019, 10, 131, doi:10.1038/s41419-018-1188-3.

19. Kobayashi, A. Roles of NRF3 in the Hallmarks of Cancer: Proteasomal Inactivation of Tumor Suppressors. Cancers 2020, 12, doi:10.3390/cancers12092681.

20. Fessler, E.; Medema, J.P. Colorectal Cancer Subtypes: Developmental Origin and Microenvironmental Regulation. Trends in cancer 2016, 2, 505-518, doi:10.1016/j.trecan.2016.07.008.

21. Wu, J.; Wang, Y.; Xu, X.; Cao, H.; Sahengbieke, S.; Sheng, H.; Huang, Q.; Lai, M. Transcriptional activation of FN1 and IL11 by HMGA2 promotes the malignant behavior of colorectal cancer. Carcinogenesis 2016, 37, 511-521, doi:10.1093/carcin/bgw029.

22. Li, Y.; Zhao, Z.; Xu, C.; Zhou, Z.; Zhu, Z.; You, T. HMGA2 induces transcription factor Slug expression to promote epithelial-to-mesenchymal transition and contributes to colon cancer progression. Cancer letters 2014, 355, 130-140, doi:10.1016/j.canlet.2014.09.007.

23. Mughees, M.; Sengupta, A.; Khowal, S.; Wajid, S. Mechanism of tumour microenvironment in the progression and development of oral cancer. Molecular biology reports 2021, 48, 1773-1786, doi:10.1007/s11033-020-06054-6.

24. Liu, H.; Mei, F.C.; Yang, W.; Wang, H.; Wong, E.; Cai, J.; Toth, E.; Luo, P.; Li, Y.M.; Zhang, W.; et al. Epac1 inhibition ameliorates pathological angiogenesis through coordinated activation of Notch and suppression of VEGF signaling. Science advances 2020, 6 , eaay3566, doi:10.1126/sciadv.aay3566.

25. Acevedo, L.M.; Barillas, S.; Weis, S.M.; Gothert, J.R.; Cheresh, D.A. Semaphorin 3A suppresses VEGF-mediated angiogenesis yet acts as a vascular permeability factor. Blood 2008, 111, 2674-2680, doi:10.1182/blood-2007-08-110205.

26. Shi, Z.; Li, X.; Wu, D.; Tang, R.; Chen, R.; Xue, S.; Sun, X. Silencing of HMGA2 suppresses cellular proliferation, migration, invasion, and epithelial-mesenchymal transition in bladder cancer. Tumour biology: the journal of the International Society for Oncodevelopmental Biology and Medicine 2016, 37, 7515-7523, 
doi:10.1007/s13277-015-4625-2.

27. Meirson, T.; Gil-Henn, H.; Samson, A.O. Invasion and metastasis: the elusive hallmark of cancer. Oncogene 2020, 39, 2024-2026, doi:10.1038/s41388-019-1110-1.

28. Liu, K.; Mou, Y.; Shi, X.; Liu, T.; Chen, Z.; Zuo, X. Circular RNA 100146 Promotes Colorectal Cancer Progression by the MicroRNA 149/HMGA2 Axis. Molecular and cellular biology 2021, 41, doi:10.1128/MCB.00445-20.

29. Poleszczuk, J.; Hahnfeldt, P.; Enderling, H. Therapeutic Implications from Sensitivity Analysis of Tumor Angiogenesis Models. PloS one 2015, 10, doi:ARTN e0120007

10.1371/journal.pone.0120007.

30. Ferrara, N.; Hillan, K.J.; Gerber, H.P.; Novotny, W. Discovery and development of bevacizumab, an anti-VEGF antibody for treating cancer. Nature reviews. Drug discovery 2004, 3, 391-400, doi:10.1038/nrd1381.

31. Berretta, M.; Lleshi, A.; Zanet, E.; Bearz, A.; Simonelli, C.; Fisichella, R.; Nasti, G.; Berretta, S.; Tirelli, U. Bevacizumab plus irinotecan-, fluorouracil-, and leucovorin-based chemotherapy with concomitant HAART in an HIV-positive patient with metastatic colorectal cancer. Onkologie 2008, 31, 394-397, doi:10.1159/000132360.

32. Miller, K.; Wang, M.; Gralow, J.; Dickler, M.; Cobleigh, M.; Perez, E.A.; Shenkier, T.; Cella, D.; Davidson, N.E. Paclitaxel plus bevacizumab versus paclitaxel alone for metastatic breast cancer. The New England journal of medicine 2007, 357, 2666-2676, doi:10.1056/NEJMoa072113.

33. Paz-Ares, L.; Garcia del Muro, X.; Grande, E.; Gonzalez, P.; Brosa, M.; Diaz, S. Cost-effectiveness analysis of sunitinib in patients with metastatic and/or unresectable gastrointestinal stroma tumours (GIST) after progression or intolerance with imatinib. Clin Trans/ Onco/ 2008, 10, 831-839, doi:10.1007/s12094-008-0297-3.

34. Escudier, B.; Eisen, T.; Stadler, W.M.; Szczylik, C.; Oudard, S.; Siebels, M.; Negrier, S.; Chevreau, C.; Solska, E.; Desai, A.A.; et al. Sorafenib in advanced clear-cell renal-cell carcinoma. The New England journal of medicine 2007, 356, 125-134, doi:10.1056/NEJMoa060655.

35. Lichtenberger, B.M.; Tan, P.K.; Niederleithner, H.; Ferrara, N.; Petzelbauer, P.; Sibilia, M. Autocrine VEGF Signaling Synergizes with EGFR in Tumor Cells to Promote Epithelial Cancer Development. Cel/2010, 140, 268-279, doi:10.1016/j.cell.2009.12.046. 


\section{Competing interests}

The authors declare that they have no competing interests

\section{Funding}

This study was supported by Research foundation of Chongqing Health committee (ZY201902003)

to $\mathrm{QZ}$

\section{Authors' contributions}

CCW; LY; MMT and QZ: conceive the idea; HBM; YYL; GDZ collected the patients samples and performed the IHC and XQH scored the IHC results; CCW; LY; MMT; WW conducted the most of the expertiments; RYL and MYZ performed the bio informatic analysis; GFX; ZXW; MC and QZ supervised the experiment. All authors contributed to subsequent and final drafts of this manuscript and approved the final manuscript before publication. 